\title{
A Theorem About Uniformly Converging Progressions of Constructive Functions
}

\author{
RAdOSlaV MilošEviĆ
}

\begin{abstract}
We give an example which represents an application of constructive functions in mathematical analysis, especially, to the theory of progressions. In fact, we shall prove a theorem related to the uniformly converging progression of constructive functions whose sum is not $R$-integrable on the unit interval.
\end{abstract}

\section{INTRODUCTION}

We use definitions and result given in the literature [1-7]. The terms "function" and "algorithm" that will be used here will be abbreviations for the idioms "everywhere defined constructive function" and "normal algorithm" respectively. Also, all the paragraphs about the objects which will be mentioned here are taken in the constructive sense according to the articles by N.A. Šanjin [5]. We also state some necessary definitions from B.A. Kušner [6].

Let $f$ be a function, $\delta$ an algorithm of the type $(N \rightarrow N)$, i.e. the algorithm that copies each natural number into a natural number. We shall say that $\delta$ is a rule of integrability of $f$ on $[0,1]$, if for each $n, k, l(k, l \geq 1)$ every of rational numbers:

has its double

$$
r_{0}, \ldots, r_{k-1}, r_{0}^{\prime}, \ldots, r_{1}^{\prime}
$$

$$
x_{0}, \ldots, x_{k-1}, x_{0}^{\prime}, \ldots, x_{l-1}^{\prime}
$$

such that:

$$
\begin{gathered}
r_{0}=0, \quad r_{0}<r_{1}<\cdots<r_{k}, \quad r_{k}=1, \\
r_{0}^{\prime}=0, \quad r_{0}^{\prime}<r_{1}^{\prime}<\cdots<r_{1}^{\prime}, r_{1}^{\prime}=1, \\
r_{0}=x_{0}=r_{1}=\cdots=r_{k-1}=x_{k-1}=r_{k}, \\
r_{0}^{\prime}=x_{0}^{\prime}=r_{1}^{\prime}=\cdots=r_{l-1}^{\prime}=x_{l-1}^{\prime}=r_{l}^{\prime}
\end{gathered}
$$

and

$$
\max _{0 \leq i \leq k-1}\left(r_{i+1}-r_{i}\right), \quad \max _{0 \leq j \leq l-1}\left(r_{j+1}^{\prime}-r_{j}^{\prime}\right) \cdot 2^{-\delta(n)}
$$

2000 Mathematics Subject Classification. Primary: 03F55, 04A05.

Key words and phrases. Uniformly converging, constructive functions, theory of progressions, not integrable, $R$-integral, algorithms, normal algorithms, quasi-numbers, quasi-integrable. 
i.e.

$$
\left|\sum_{i=0}^{k-1} f\left(x_{i}\right)\left(x_{i+1}-r_{i}\right)-\sum f\left(x_{i}^{\prime}\right)\left(r_{i+1}^{\prime}-r_{i}^{\prime}\right)\right|<2^{-n} .
$$

We shall say that $f$ is integrable in the sense of Riemann on $[0,1]$ (or shorter $R$-integrable) if the rule of integrability of $f$ can be applied on $[0,1]$.

Analogously, the relation "the double $x$ is Riemann's integral ( $R$-integral) of the function $f$ on $[0,1]$ " can be introduced. Using, the rule of the integrability of a given function it is not difficult to obtain the double over the integral.

The function $f$ is uniformly continuous on the interval $[a, b]$ if it is possible to construct an algorithm $\delta$ of type $(N \rightarrow N)$, which is called the rule of the uniform continuity of $f$ on $[a, b]$, such that for each $n, x_{1}, x_{2}$ with $x_{1}, x_{2} \in[a, b]$ and

$$
\left|x_{1}-x_{2}\right|<2^{-\delta(n)}
$$

the following inequality is satisfied:

$$
\left|f\left(x_{1}\right)-f\left(x_{2}\right)\right|<2^{-n} .
$$

We shall say that $f$ is effectively non-uniformly continuous on $[a, b]$, if it is possible to find a natural number $l$ and algorithms $\alpha, \beta$ such that:

1) $\alpha$ and $\beta$ convert every natural number in a double from $[a, b]$,

2) for each $n,|\alpha(n)-\beta(n)|<2^{-n}$ and $|f(\alpha(n))-f(\beta(n))| \geq 2^{-l}$ hold.

The function $f$ is everywhere effectively non-uniformly continuous on $[0,1]$ if it is effectively non-uniformly continuous on each non-degenerative interval that is included in $[0,1]$.

An algorithm $F$ will be called the series of functions if for each $n$ the algorithm $F$ is a function. A function $f$ will be called quasi-integrable on $[0,1]$ if it is not true that it is not $R$-integrable on $[0,1]$. Let $F$ is a series of functions. We shall consider the functional progression

$$
\sum_{i=1}^{\infty} F_{i}(x),
$$

which will mean that the algorithm $T$ was built in the previously determined way, such that for each $n$ and $x$ :

$$
T(n, x)=\sum_{i=0}^{n} \tilde{F}_{i}(x) .
$$

An algorithm $\delta$ of the type $(N \rightarrow N)$ will be called the rule of uniform convergence of the progression (1) on the sets of doubles $M$, if for each $k, l, n$, and $x$, such that $l \geq \delta(k)$ and $x \in M$, the following condition is satisfied:

$$
\left|\sum_{i=l}^{l+m} \tilde{F}_{i}(x)\right|<2^{-k}
$$


We shall say that functional progression (1) is uniformly convergent on $M$, if it is possible to formulate the rule of uniform convergence of that progression on $M$.

We shall assume now that the progression (1) uniformly converges on $[0,1]$ and that all the functions $F_{i}$ are $R$-integrable on $[0,1]$. This means that it is possible to build an algorithm $\alpha$ such that for each $i$ the algorithm $\tilde{\alpha}_{i}$ is the rule of integrability of $F_{i}$ on $[0,1]$. It is not difficult to see that when such an algorithm $\alpha$ and the rule of the uniform convergence of progression (1) are available, it is possible to formulate the rule of integrability on $[0,1]$. Thus, the sum of the functional progression which uniformly converges on $[0,1]$, whose terms are $R$ integrable, is also $R$-integrable on $[0,1]$.

The next theorem will show that only with such a presentation the condition of the $R$-integrability of function $F_{i}$ can not be weaken.

Theorem. It is possible to build the series of functions $G$ such that all the functions $\tilde{G}_{i}$ are quasi-integrable on $[0,1]$, and the functional progression $\sum_{i=0}^{\infty} G_{i}$ uniformly converges along the complete axis, so that the function as the sum is not $R$-integrable on $[0,1]$ according to Riemann.

Before the proof of the theorem we shall state two lemmas:

Lemma 1. It is possible to build an algorithm $\alpha$ such that for each quasi-number $q$ and natural number $n$ with $|q|<2^{-n}$, there exists a function $\tilde{\alpha}_{q, n}$ such that for each $x$ we have:

$$
\left|\tilde{\alpha}_{q, n}(x)\right|<2^{-n+7}
$$

and $q$ is the conditionally $R$-integrable function $\tilde{\alpha}_{q, n}$ on $[0,1]^{1}$.

This lemma can be proved by insignificant modification of the construction that is indicated in the proof of the Theorem 2 in [6]. We can conclude from Lemma 1 and the obvious circumstance that from each $R$-integrable function it is possible to obtain an $F$-number which is associated with its integral; thus the theorem about the completeness of $F$-number can be formulated without difficulties $[5,6]$.

Lemma 2. It is possible to build a series of quasi-numbers $\beta$ such that for each $m \geq n$ it holds:

$$
|\beta(m)-\beta(n)|<2^{-n}
$$

and the series $b$ does not converge to any double.

This statement is given without proof in [4]. We give one of its possible proofs. Let $S$ be a series of rational numbers such that for each $n$

$$
0<S(n) \leq S(n+1)<1
$$

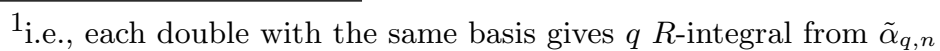


and the series $S$ does not converge to any double. We shall build the algorithm $T$ such that:

$$
\begin{aligned}
T(k, 0) & =S(0) ; \\
T(k, l+1) & =\left\{\begin{array}{lll}
T(k, l), & \text { if } & S(l+1)-T(k, l)<2^{-k-1} \\
S(l+1), & \text { if } & S(l+1)-T(k, l) \geq 2^{-k-1} .
\end{array}\right.
\end{aligned}
$$

Further, let us build the algorithm $\beta$ such that for each $k$ :

$$
\beta(k)=\tilde{T}_{k} .
$$

It is not difficult to see that $\beta$ is the required series of quasi-numbers.

Proof of the theorem. Let $\beta$ be the construction containing only the series of quasinumbers. Let us build the algorithm $\gamma$ such that:

$$
\gamma(o)=\beta(o) ; \quad \gamma(n+1)=\beta(n+1)-\beta(n) .
$$

It is obvious that for $n \geq 1$ the inequality $|\gamma(n)|<2^{-n}$ is satisfied. Applying the lemmas we can build the algorithm $G$ satisfying the following conditions:

1) $G$ is a series of functions;

2) for each $i$ the quasi-number $\gamma(i)$ is conditionally $R$-integral $\tilde{G}_{i}$ on $[0,1]$;

3) for $i \geq 1$ and each $x$ it is satisfied:

$$
\left|\tilde{G}_{i}(x)\right|<2^{-i+7} \text {. }
$$

By characteristic 3) of the algorithm $G$, the functional progression $\sum_{i=0}^{\infty} G_{i}$ uniformly converges along the complete axis. If that function would be $R$-integrable on $[0,1]$ then there would be a double as $R$-integrable $f$ on $[0,1]$. Then, what is not difficult to see, the series of quasi-numbers would converge towards that double, which is not possible. Consequently, it follows that $f$ is not $R$-integrable on $[0,1]$, what we wanted to show.

We shall state now several obvious consequences of this theorem.

Consequence 1. There is no any algorithm which would transform each $n$ into the rule of integrability of the function $\tilde{G}_{n}$ on $[0,1]$.

Consequence 2. There is no such an algorithm which would transform each $n$ into a double that would be the $R$-integral of $G_{n}$ on $[0,1]$.

Consequence 3. There is no algorithm which transforms each $R$-integrable function on $[0,1]$ into its rule of integrability on $[0,1]$.

Consequence 4. There is no such an algorithm that would transform each function which is $R$-integrable on $[0,1]$ into a double as an $R$-integral of the function on $[0,1]$. 


\section{REFERENCES}

[1] A.N. Kolmogorov, Tri podhoda $k$ opredeleniju ponjatija "količestvo informacii", "Problemy peredači informacii", No. 1 (1965), 3-11.

[2] A.A. Markov, O konstruktivnoj matematike, Tr. Matem. in-ta im. V. A. Steklova, 1962, t. XXII, 8-14.

[3] P. Peter, Rekurzivnye funkcii. M., Izd-vo in. lit., 1954.

[4] N.A. Šanjin, Konstruktivnye vešćestvennye čisla i konstruktivnye funkcionaklnye prostranstva, Tr. Matem. in-ta im V.A. Steklova, 1962, t. LXXII, 15-294.

[5] N.A. Šanjin, O konstruktivnom poimanii matematičeskih suidenij, Tr. Matem. in-ta im. V. A. Steklova, 1958, t. LII, 226-311.

[6] B.A. Kušner, Lekcii po konstruktivnomu matematičeskomu analizu, M. Nauka, 1973.

[7] S.K. Klini, Vvedenie v matematiku, K.M., Izd-vo in. lit., 1957.

FilOzOfSKi FAKULTET (ODSJEK MATEMATIKA)

Alekse Šantica BB

Istocno SARAJEVo (PALE)

RePUblika SRPSKa, BiH

E-mail address: mingeyu@yahoo.com 